\title{
Ordinary holomorphic webs of codimension one
}

\author{
VINCENT CAVALIER ${ }^{\dagger}$ AND DANIEL LEHMANN
}

\begin{abstract}
To any $d$-web of codimension one on a holomorphic $n$-dimensional manifold $M(d>n)$, we associate an analytic subset $S$ of $M$. We call ordinary the webs for which $S$ has a dimension at most $n-1$ or is empty. This condition is generically satisfied, at least at the level of germs.

We prove that the rank of an ordinary $d$-web has an upper-bound $\pi^{\prime}(n, d)$ which, for $n \geq 3$, is strictly smaller than the bound $\pi(n, d)$ proved by Chern, $\pi(n, d)$ denoting the Castelnuovo's number. This bound is optimal.

Setting $c(n, h)=\left(\begin{array}{c}n-1+h \\ h\end{array}\right)$, let $k_{0}$ be the integer such that $c\left(n, k_{0}\right) \leq d<c\left(n, k_{0}+1\right)$. The number $\pi^{\prime}(n, d)$ is then equal

$$
\begin{aligned}
& \text { - to } 0 \text { for } d<c(n, 2), \\
& \text { - and to } \sum_{h=1}^{k_{0}}(d-c(n, h)) \text { for } d \geq c(n, 2) .
\end{aligned}
$$
\end{abstract}

Moreover, if $d$ is precisely equal to $c\left(n, k_{0}\right)$, we define off $S$ a holomorphic connection on a holomorphic bundle $\mathcal{E}$ of rank $\pi^{\prime}(n, d)$, such that the set of Abelian relations off $S$ is isomorphic to the set of holomorphic sections of $\mathcal{E}$ with vanishing covariant derivative: the curvature of this connection, which generalizes the Blaschke curvature, is then an obstruction for the rank of the web to reach the value $\pi^{\prime}(n, d)$.

When $n=2, S$ is always empty so that any web is ordinary, $\pi^{\prime}(2, d)=\pi(2, d)$, and any $d$ may be written $c\left(2, k_{0}\right)$ : we recover the results given in [9].

Mathematics Subject Classification (2010): 53A60 (primary); 14C21, 32S65 (secondary).

\section{Introduction}

A holomorphic $d$-web $W$ of codimension one on a $n$-dimensional holomorphic manifold $M$ being given $(d>n)$, we denote by $M_{0}$ the open set in $M$ where the web is locally defined by $d$ holomorphic foliations $\mathcal{F}_{i}$ of codimension one, all non-singular and with tangent spaces to the leaves distinct at any point.

We shall say that the web is in strong general position (SGP) if any subset of $n$ leaves among the $d$ leaves through a point of $M_{0}$ are in general position. For

Vincent Cavalier passed away on July $22^{\text {nd }}, 2010$.

Received March 24, 2010; accepted in revised form November 25, 2010. 
instance, any algebraic web (i.e. any web in $\mathbb{P}_{n}$ whose leaves are the tangent hyperplanes belonging to some irreducible non-degenerate algebraic curve in the dual projective space $\mathbb{P}_{n}^{\prime}$ ) is SGP. If we assume only that there exists a subset of $n$ leaves among the $d$ 's which are in general position (but not necessarily any $n$ of them), we shall say that the web is in weak general position (WGP). Most of our results below will require only this weaker condition ${ }^{1}$. Notice that, for a WGP web which is not SGP, the various local foliations defining the web will not all play the same role.

On an open set $U$ in $M_{0}$ sufficiently small for the web to be defined as above by the data of $d$ distinct local foliations $\mathcal{F}_{i}$ (such an open set is called "open set of distinguishability"), an Abelian relation is the data of a family $\left(\omega_{i}\right)_{i}$ of holomorphic 1 -forms $\omega_{i},(1 \leq i \leq d)$ such that

(i) each $\omega_{i}$ is closed,

(ii) the vector fields tangent to the local foliation $\mathcal{F}_{i}$ belong to the kernel of $\omega_{i}$,

(iii) the sum $\sum_{i=1}^{d} \omega_{i}$ vanishes.

[If $U$ is moreover simply connected, each $\omega_{i}$ of such an Abelian relation has a holomorphic primitive $f_{i}$ which is a first integral of the local foliation $\mathcal{F}_{i}$. The definition above of an Abelian relation is therefore equivalent -at the level of germsto the original one which was: "a family $\left(f_{i}\right)$ of first integrals for the local foliations $\mathcal{F}_{i}$ defining the web, defined up to an additive constant, such that $\sum_{i} f_{i}$ be constant".]

The set of Abelian relations on $U$ (respectively the set of germs of Abelian relation at a point $m$ of $M_{0}$ ) has the structure of a finite dimensional complex vector space, whose dimension is called the rank of the web on $U$ (respectively at $m$ ). For a SGP web, Hénaut proved that its rank at a point does not depend on this point [10]: Abelian relations have then the structure of a local system of coefficients ${ }^{2}$. When we require only the web to be WGP, we shall call "rank of the web" the maximum of the rank at each point of $M_{0}$. Notice that, in this case, the rank at a point is an upper-semicontinuous function of the point.

The terminology "Abelian relation" arises from a theorem of Abel which asserts (in another language, and after duality) that the rank of the algebraic $d$-web in $\mathbb{P}_{n}$, whose leaves are the hyperplanes belonging to some irreducible non-degenerate algebraic curve $\Gamma$ of degree $d$ in the dual projective space, is at least equal to the arithmetical genus of $\Gamma$.

On the other hand, Chern proved that the rank of a SGP web is always upperbounded by the so-called Castelnuovo's number

$$
\pi(n, d)=\sum_{h \geq 1}(d-h(n-1)-1)^{+}, \text {where }(a)^{+}=\sup (a, 0),
$$

\footnotetext{
${ }^{1}$ More generally, if there exists $\ell$ and not more among the leaves through a point which are in general position $(\ell \leq n)$, there exists locally a holomorphic foliation $\mathcal{G}$ of codimension $\ell$, such that the web is locally the pullback of a $d$-web of codimension 1 on a $\ell$-dimensional manifold transverse to $\mathcal{G}$. Then, many of the results below would remain valid, after replacing $n$ by $\ell$.

2 In [11], he generalized this result in higher codimension.
} 
that Castelnuovo proved to be the maximum of the arithmetical genus of an irreducible non-degenerate algebraic curve of degree $d$ in the $n$-dimensional complex projective space $\mathbb{P}_{n}$ (see [8]). Combining these theorems of Abel, Castelnuovo and Chern, we deduce that the bound $\pi(n, d)$ is optimal for SGP webs.

The non-algebraizable or non-linearizable webs which have maximal rank $\pi(n, d)$ are usually called exceptional. The first example of such a web has been given by Bol seventy years ago, but many among the more or less recent works, including those by Chern-Griffiths, Goldberg-Lychagin, Hénaut, Pereira, Pirio, Robert, Trépreau,... discuss problems around this subject. See surveys with many historical references in Hénaut [12], Pereira [14], and Pereira-Pirio [16].

We worked in another direction. In fact, given a $d$-web on a holomorphic surface, Hénaut constructed in [9] a holomorphic bundle of rank $\pi(2, d)=(d-1)(d-2) / 2$ above the surface, with a holomorphic connection $\nabla$, such that Abelian relations may be identified with holomorphic sections $u$ of this bundle having a vanishing covariant derivative $(\nabla u=0)$ : the curvature of this connection is therefore an obstruction for the web to have maximal rank $\pi(2, d)$, generalizing the BlaschkeDubourdieu curvature of the case $d=3$. The method (whose principle is already in an old paper of Pantazi [13] but not using connections) consists in the construction of some prolongation of the partial derivative equation, the solutions of which are the Abelian relations.

The first aim of our work was to generalize this construction of Hénaut to any $n \geq 2$. But some difficulties appear which do not exist in dimension 2. Lemma 2.2 below implies in fact that two conditions must be satisfied:

- on one hand, the construction may work only off some analytical subset $S$ of $M_{0}$, attached to the web, and has therefore little interest if $S$ has the same dimension $n$ as $M$;

- on the other hand, $d$ must be equal, for some $h \geq 2$, to the dimension $c(n, h)$ of the vector space of all homogeneous polynomials of degree $h$ in $n$ variables with scalar coefficients).

In the case $n=2$, it happens that $S$ is always empty, and that any $d$ may be written $c(2, d-1)$.

But, for $n \geq 3, S$ may be non-empty; by chance, if it is not empty, it has generically (at least locally) a dimension at most $n-1$ : it is the reason why we shall say below that such webs are ordinary. Rather than "extraordinary", the other ones will be said to be special. For example, a foliation defined by a pencil of parallel planes in $\mathbb{C}^{3}$ is determined by the common line of these planes at infinity; thus, given six such lines in the plane at infinity, they define a 6-web in $\mathbb{C}^{3}$. Such a web is ordinary as far as the six lines are not tangent to a same conic: this condition is obviously generically satisfied.

By the way, we proved that, whatever be $d(d>n)$, not necessarily belonging to the previous sequence $(c(n, h))_{h}$, the rank of any ordinary $d$-web is at most equal to some bound $\pi^{\prime}(n, d)$ which, for $n \geq 3$, is strictly smaller than the Chern's bound $\pi(n, d)$ by the Castelnuovo's number. 
More precisely, for any $d,(d>n)$, denote by $k_{0}$ the integer $(\geq 1)$ such that

$$
c\left(n, k_{0}\right) \leq d<c\left(n, k_{0}+1\right),
$$

and set:

$$
\begin{array}{rlrl}
\pi^{\prime}(n, d) & = & 0 & \text { when } d<c(n, 2),\left(k_{0}=1\right), \\
& =\sum_{h=1}^{k_{0}}(d-c(n, h)) & \text { when } d \geq c(n, 2),\left(k_{0} \geq 2\right) .
\end{array}
$$

The main results of this paper are then the two following:

Theorem 1.1. The rank of any ordinary $d$-web on some $n$-dimensional manifold $M_{0}$ is at most equal to $\pi^{\prime}(n, d)$. This bound is optimal.

Theorem 1.2. If $d=c\left(n, k_{0}\right)$, and if the $d$-web is ordinary, there exists a holomorphic vector bundle $\mathcal{E}$ of rank $\pi^{\prime}(n, d)$ over $M_{0} \backslash S$ and a holomorphic connection $\nabla$ on $\mathcal{E}$, such that the vector space of germs of Abelian relations at a point of $M_{0} \backslash S$ is isomorphic to the vector space of germs of sections of $\mathcal{E}$ which have a vanishing covariant derivative : the curvature of this connection is then an obstruction for the rank of the web to reach the maximal value $\pi^{\prime}(n, d)$.

Remark 1.3. Unfortunately, this curvature is often difficult to compute manually. It would be very interesting to perform a good program providing easily this curvature by computer.

In the last section, we give a second proof of theorem 1-1 in the particular case of SGP webs. We prove also that all ordinary parallel $d$-webs in $\mathbb{C}^{n}$ (i.e. ordinary $d$-webs which are defined by $d$ pencils of parallel hyperplanes) have rank $\pi^{\prime}(n, d)$, hence the optimality of this bound. We give finally significative examples of the various possible situations.

We have announced our results in a preprint (arXiv [4]), and a survey without proof has been published in [5] (where we called "regular" the webs that we say now to be "ordinary"). We tried also to give a global meaning to our constructions, using our paper [6] (generalizing partially [7]).

The notion of "ordinary web" induces obviously problems in algebraic geometry, that we hope to study in a near future:

- what are the "ordinary irreducible non-degenerate algebraic curves" in $\mathbb{P}_{n}$ (i.e. curves whose associated web in the dual projective space is ordinary)?

- among the ordinary curves of degree $d$, which ones have maximal arithmetical genus $\pi^{\prime}(n, d)$ ?

- what can be said about the algebraizability of ordinary webs having "sufficiently many" Abelian relations? (see $[16,19]$ for the non-necessarily ordinary case).

Acknowledgements. We thank A. Hénaut, J.V. Pereira and L. Pirio for very helpful conversations. 


\section{Notations and backgrounds}

Most of the results in this section are well known or easy to prove, so that we shall omit their proof.

\subsection{Some algebraic notations}

Let $\mathbb{R}_{h}\left[X_{1}, \cdots, X_{n}\right]$ denote the vector space of homogeneous polynomials of degree $h$ in $n$ variables, with scalar coefficients (in fact, the field of scalars does not matter). Denote by $c(n, h)$ its dimension $\left(\begin{array}{c}h+n-1 \\ h\end{array}\right)$. The monomials $X^{L}=\left(X_{1}\right)^{\lambda_{1}} \cdots\left(X_{n}\right)^{\lambda_{n}}$ make a basis, indexed by the set $\mathcal{P}(n, h)$ of the partitions $L=\left(\lambda_{1}, \lambda_{2}, \cdots, \lambda_{n}\right)$ of $h$, with $0 \leq \lambda_{i} \leq h$ for any $i=1, \cdots, n$, and $\sum_{i=1}^{n} \lambda_{i}=h$. The number $h$ will be also denoted by $|L|$, and is called the height of $L$.

Denoting by $(a)^{+}$the number sup $(a, 0)$ for any real number $a$, remember $([8])$ that the number

$$
\pi(n, d)=\sum_{h \geq 1}(d-h(n-1)-1)^{+},
$$

called the Castelnuovo's number, is the maximum of the arithmetical genus of an algebraic irreducible non-degenerate curve of degree $d$ in the complex projective space $\mathbb{P}_{n}$.

Define

$$
\begin{array}{rlrl}
\pi^{\prime}(n, d) & = & 0 & \text { when } d<c(n, 2), \\
& =\sum_{h \geq 1}(d-c(n, h))^{+} & \text {when } d \geq c(n, 2) .
\end{array}
$$

\section{Lemma 2.1.}

(i) For $n \geq 3$, the inequality $\pi^{\prime}(n, d)<\pi(n, d)$ holds.

(ii) The equality $\pi^{\prime}(2, d)=\pi(2, d)$ holds.

\subsection{Connections adapted to a differential operator}

Let $E \rightarrow V$ be a holomorphic vector bundle on a holomorphic manifold $V$. Remember the exact sequence of holomorphic vector bundles

$$
0 \rightarrow T^{*} V \otimes E \rightarrow J^{1} E \rightarrow E \rightarrow 0,
$$

where $J^{1} E$ denotes the holomorphic bundle of 1-jets of holomorphic sections of $E$, and $T^{*} V$ the holomorphic bundle of 1 -forms of type $(1,0)$ on $V$. A $C^{\infty}$-connection $\nabla$ on $E$ of type $(1,0)$ (respectively a holomorphic connection if any) is a $C^{\infty}$. splitting (respectively a holomorphic splitting if any) of this exact sequence:

$$
0 \rightarrow T^{*} V \otimes E \stackrel{\beta}{\leftrightarrows} J^{1} E \stackrel{\alpha}{\longleftrightarrow} E \rightarrow 0 .
$$


If $u$ is a $C^{\infty}$ or holomorphic section of $E$, its covariant derivative is given by

$$
\nabla u=\beta\left(j^{1} u\right) .
$$

A holomorphic linear differential operator of order $p$ on the holomorphic manifold $V$ is a morphism of holomorphic vector bundles $D: J^{p} E \rightarrow F$, for some vector bundles $E$ and $F$ on $V$, to which we associate the map $\mathcal{D}: u \mapsto D\left(j^{p} u\right)$ from the sections of $E$ into the sections of $F$. The kernel $R$ of $D$ is a subset of $J^{p} E$, which is the set of the formal solutions at order $p$ of the equation $\mathcal{D} u=0$. A section $u$ of $E$ such that $\mathcal{D} u=0$ is called a "solution" of this equation

The morphism $D$ induces a morphism $j^{1} D: J^{1}\left(J^{p} E\right) \rightarrow J^{1} F$ whose restriction $\widetilde{D}: J^{p+1} E \rightarrow J^{1} F$ to the sub-bundle $J^{p+1} E \subset J^{1}\left(J^{p} E\right)$ is called the first prolongation of $D$, and the sections $u$ of $E$ such that $\widetilde{D}\left(j^{p+1} u\right)=0$ are also the solutions of the equation $\mathcal{D} u=0$.

Assume that $R$ is a holomorphic vector bundle above $V$. Then $J^{1} R$ is a subvector bundle of $J^{1}\left(J^{p} E\right)$, as well as $J^{p+1} E$. The kernel $R^{\prime}$ of the morphism $\widetilde{D}: J^{p+1} E \rightarrow J^{1} F$ (the space of formal solutions at order $p+1$ of the equation $\mathcal{D} u=0)$ is then equal to the intersection $J^{1} R \cap J^{p+1} E$ in $J^{1}\left(J^{p} E\right)$.

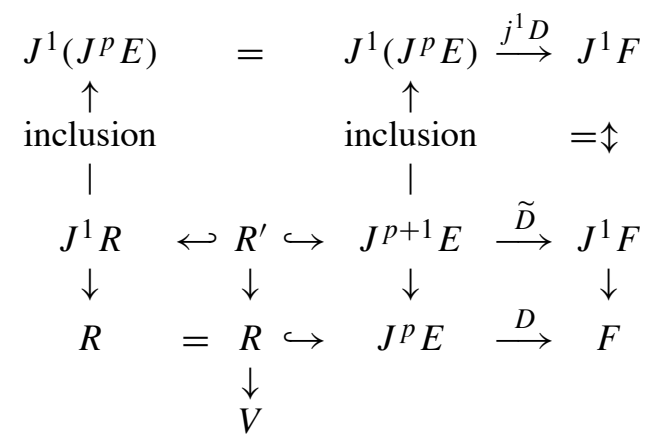

From the Spencer-Goldschmidt theory [18], we deduce:

Lemma 2.2. The two following assertions are equivalent:

(i) The projection $R^{\prime} \rightarrow R$ is an isomorphism of holomorphic vector bundles.

(ii) There is a holomorphic connection $\nabla$ on $R \rightarrow V$ such that the map $u \mapsto j^{p} u$ is an isomorphism from the space of solutions $u$ of the equation $\mathcal{D} u=0$ into the space of sections of $R$ with vanishing covariant derivative.

Moreover, such a connection $\nabla$ is unique and defined as being the map $\alpha: R \rightarrow J^{1} R$ equal to the composition of the inclusion $R^{\prime} \hookrightarrow J^{1} R$ with the inverse isomorphism $R \rightarrow R^{\prime}$.

[This lemma is used by Hénaut in [9] under an equivalent form.] 


\subsection{The differential operator for Abelian relations}

Recall (see [6]) that, globally, a WGP $d$-web on a $n$-dimensional holomorphic manifold $M$ (with $d>n$ ) is defined by a $n$-dimensional analytical subspace $W$ of the projectivized cotangent space $\mathbb{P}\left(T^{*} M\right)$, on the smooth part of which the canonical contact form becomes integrable and induces a foliation $\widetilde{\mathcal{F}}]$. Let $W_{0}$ be the set of points in $W$ over $M_{0}$ and $\pi_{W}: W_{0} \rightarrow M_{0}$ be the $d$-fold corresponding covering. For any complex holomorphic vector bundle $E$ of rank $r$ over $W_{0}$, let $\pi_{*} E$ be the holomorphic bundle of rank $d \times r$ over $M_{0}$, whose fiber at a point $m \in M_{0}$ is defined by

$$
\left(\pi_{*} E\right)_{m}=\bigoplus_{\tilde{m} \in\left(\pi_{W}\right)^{-1}(m)} E_{\widetilde{m}} .
$$

The sheaf of holomorphic sections of $\pi_{*} E$ is then the direct image of the sheaf of holomorphic sections of $E$ by $\pi_{W}$. For instance, a germ $\omega$ of $\pi_{*}\left(T^{*} \widetilde{\mathcal{F}}\right)$ at $m$ is a family $\left(\omega_{i}\right)$ of germs $\omega_{i}$ at $m$ of forms $(1 \leq i \leq d)$, such that the kernel of $\omega_{i}$ contains the tangent space $T_{i}$ to the local foliation $\mathcal{F}_{i}$ (and is therefore equal to it when $\omega_{i}$ is not zero).

Let $\operatorname{Tr}: \pi_{*}\left(T^{*} \widetilde{\mathcal{F}}\right) \rightarrow T^{*} M_{0}$ be the morphism of holomorphic vector bundles given by

$$
\operatorname{Tr} \omega=\sum_{i=1}^{d} \omega_{i} .
$$

Observe that this morphism doesn't depend on the numbering of the $\omega_{i}$, since $\sum_{i}$ is symmetric with respect to the indices $i$, so that $\operatorname{Tr}$ has a global meaning. Since the web is WGP, it is easy to check that Tr has constant rank $n$. Then, we define a holomorphic vector bundle $A$ of rank $d-n$ over $M_{0}$ as the kernel of this morphism.

Definition 2.3. The vector bundle $A$ of rank $d-n$ so defined will be called the Blaschke bundle of the web.

Let us define similarly a holomorphic vector bundle $B$ over $M_{0}$, of rank $(d-1) n(n-1) / 2$, as the kernel of the morphism $\operatorname{Tr}: \pi_{*}\left(\bigwedge^{2} T^{*} W_{0}\right) \rightarrow \bigwedge^{2} T^{*} M_{0}$ given locally by $\operatorname{Tr} \varpi=\sum_{i=1}^{d} \varpi_{i}$.

Definition 2.4. We call Abelian relation of the web any holomorphic section $u$ of the Blaschke bundle $A$, which is solution of the equation $\mathcal{D} u=0$, the map $\mathcal{D}$ denoting the linear first order differential operator from $A$ to $B$ locally defined by $\left(\omega_{i}\right) \mapsto\left(d \omega_{i}\right)$.

The differential operator $\mathcal{D}$ may still be seen as a linear morphism $D: J^{1} A \rightarrow B$, and the kernel $R_{0}$ of this morphism is the space of "formal Abelian relations at order one". Hence, a necessary condition for an Abelian relation to exist above an open subset $U$ of $M_{0}$ is that $U$ belongs to the image of $R_{0}$ by the projection $J^{1} A \rightarrow A$. We shall see that it generically not the case for $d<c(n, 2)$.

More generally, denote by $D_{k}: J^{k+1} A \rightarrow J^{k} B$ the $k^{\text {th }}$ prolongation of $D$. The kernel $R_{k}$ of the morphism $D_{k}$ is the space of formal Abelian relations at order 
$k+1$. Abelian relations may still be seen as the holomorphic sections $u$ of $A$ such that $j^{k+1} u$ be in $R_{k}$. Let $\pi_{k+1}$ denote the natural projection $R_{k+1} \rightarrow R_{k}$. Let $\sigma_{k+1}: S^{k+2}\left(T^{*} M_{0}\right) \otimes A \rightarrow S^{k+1}\left(T^{*} M_{0}\right) \otimes B$ be the symbol of $D_{k+1}, g_{k+1}$ be the kernel of this symbol, and $K_{k}$ its cokernel. After the snake's lemma, there is a natural map $\partial_{k}: R_{k} \rightarrow K_{k}$ in such a way that we get a commutative diagram with all lines and columns exact.

[Notice that, in this diagram, the $R_{k}$ 's, $g_{k}$ 's and $K_{k}$ 's are not necessarily vector bundles: exactness has then to be understood as the exactness on the fibers at any point of $M_{0}$. Moreover, we allow $k$ to take the value -1 , with the convention $\left.R_{-1}=A, J^{-1} B=0.\right]$

\begin{tabular}{|c|c|c|c|c|c|c|}
\hline & 0 & & 0 & & 0 & $R_{k}$ \\
\hline & $\downarrow$ & & $\downarrow$ & & $\downarrow$ & $\downarrow \partial_{k}$ \\
\hline $0 \rightarrow$ & $g_{k+1}$ & $\rightarrow S$ & ${ }^{-2}\left(T^{*} M_{0}\right)$ & $\stackrel{\sigma_{k+1}}{\longrightarrow}$ & $S^{k+1}\left(T^{*} M_{0}\right) \otimes B$ & $K_{k} \quad \rightarrow 0$ \\
\hline & $\downarrow$ & & $\downarrow$ & & $\downarrow$ & $\downarrow$ \\
\hline $0 \rightarrow$ & $R_{k+1}$ & $\rightarrow$ & $J^{k+2} A$ & $\stackrel{D_{k+1}}{\longrightarrow}$ & $J^{k+1} B$ & $\rightarrow$ coker $D_{k+1} \rightarrow 0$ \\
\hline & $\downarrow \pi_{k+1}$ & & $\downarrow$ & & $\downarrow$ & $\downarrow$ \\
\hline$\rightarrow$ & $R_{k}$ & $\rightarrow$ & $J^{k+1} A$ & $\stackrel{D_{k}}{\longrightarrow}$ & $J^{k} B$ & $\rightarrow$ coker $D_{k} \rightarrow 0$ \\
\hline & $\downarrow \partial_{k}$ & & $\downarrow$ & & $\downarrow$ & $\downarrow$ \\
\hline & $K_{k}$ & & 0 & & 0 & 0 \\
\hline
\end{tabular}

In the sequel,

the index $i$ will run from 1 to $d$,

the indices $\alpha, \beta, \cdots$ will run from 1 to $n-1$,

and the indices $\lambda, \mu, \cdots$ will run from 1 to $n$.

For any holomorphic function $a$ and local coordinates $x, a_{\lambda}^{\prime}$ will denote the partial derivative $a_{\lambda}^{\prime}=\frac{\partial a}{\partial x_{\lambda}}$. More generally, for any partition $L=\left(\lambda_{1}, \lambda_{2}, \cdots, \lambda_{n}\right)$ of $|L|=\sum_{\mu} \lambda_{\mu},(L \in \mathcal{P}(n,|L|))$, we denote by $(a)_{L}^{\prime}$ the corresponding higher

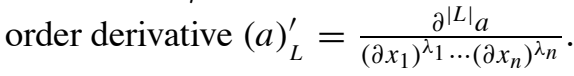

\section{Definition of the analytical set $S$}

This definition is different according to the inequalities $d<c(n, 2)$ or $d \geq c(n, 2)$. However, in both cases, this set will satisfy to the 
Lemma 3.1. The set $S$ is an analytical set which, at the level of germs, has generically a dimension $\leq n-1$ or is empty.

\subsection{Definition of $S$ in the case $n<d<c(n, 2)$}

For $n<d<c(n, 2), S$ will denote the subset of elements $m \in M_{0}$ such that the vector space $\left(R_{0}\right)_{m}=\left(\pi_{0}\right)^{-1}\left(A_{m}\right)$ has dimension at least 1 . The proof of Lemma 3.1 for $d<c(n, 2)$ will be given in the next section, after the description of the map $\pi_{0}$.

Proof of Theorem 1.1 in the case $d<c(n, 2)$. For $d<c(n, 2)$, all ordinary $d$-webs of codimension one have rank 0 .

The fact that all ordinary $d$-webs have rank 0 for $d<c(n, 2)$ on $M_{0} \backslash S$ is a tautology, because of the definition of $S$. Therefore, they still have rank 0 on all of $M_{0}$, because of the semi-continuity of the rank at a point.

\subsection{Definition of $S$ in the case $d \geq c(n, 2)$}

Let $\eta_{i}$ be an integrable 1-form defining the local foliation $\mathcal{F}_{i}$ of a $d$-web of codimension 1 . For any integer $h \geq 1$, let $\left(\eta_{i}\right)^{h}$ be the $h^{\text {th }}$ symetric power of $\eta_{i}$ in the space $S^{h}\left(T^{*} M_{0}\right)$ of homogeneous polynomials of degree $h$ on the complex tangent tangent bundle $T M_{0}$. For any $m \in M_{0}$, let $r_{h}(m)$ be the dimension of the subspace $L_{h}(m)$ generated in $S^{h}\left(T_{m}^{*} M_{0}\right)$ by the $\left(\eta_{i}\right)^{h}(m)$ 's with $1 \leq i \leq d$ (not depending on the choice of the $\eta_{i}$ 's). We have obviously the

Lemma 3.2. The following inequality holds:

$$
r_{h}(m) \leq \min (d, c(n, h)) .
$$

In particular, $r_{1} \equiv n$, since we assume $d>n$ and the web to be WGP. And $r_{h} \equiv d$ for $h>k_{0}$, where $k_{0}$ denotes the integer such that

$$
c\left(n, k_{0}\right) \leq d<c\left(n, k_{0}+1\right) .
$$

Definition 3.3 (of $S$ for $d \geq c(n, 2)$ (i.e. $\left.k_{0} \geq 2\right)$ ). Let $S_{h}$ be the set of points $m \in M_{0}$ such that $r_{h}(m)<\min (d, c(n, h))$, and set: $S=\bigcup_{h=2}^{k_{0}} S_{h}$.

Proof of Lemma 3.1 for $d \geq c(n, 2)$. Let $\left(x_{\lambda}\right)_{1 \leq \lambda \leq n}$ be a system of holomorphic local coordinates near a point $m_{0}$ of $M_{0}$, and assume that the local foliation $\mathcal{F}_{i}$ is defined by $\sum_{\lambda} p_{i \lambda} d x_{\lambda}=0$. Then $r_{h}(m)$ is the rank of the matrix

$$
P_{h}=\left(\left(C_{i L}^{(h)}\right)\right)_{i, L}
$$

of size $d \times c(n, h)$ at point $m$, where $1 \leq i \leq d$, and $L$ runs through the set $\mathcal{P}(n, h)$ of the partitions $L=\left(\lambda_{1}, \lambda_{2}, \cdots, \lambda_{n}\right)$ of $h\left(i . e . \sum_{s=1}^{n} \lambda_{s}=h\right)$, and where $C_{i L}^{(h)}=\prod_{s=1}^{n} p_{i \lambda_{s}}$. Thus, for $2 \leq h \leq k_{0}, S_{h}$ is locally defined by the vanishing of all determinants of size $c(n, h)$ in $P_{h}$. Exceptionnaly, it may happen that all of these determinants are identically 0 , so that $S_{h}$ will have dimension $n$. But generically, these determinants will vanish on hypersurfaces or nowhere. 


\section{Computation of $R_{0}$}

Locally, the $d$-web is defined over $M_{0}$ by a family of 1 -forms

$$
\eta_{i}=d x_{n}-\sum_{\alpha} p_{i \alpha}(x) d x_{\alpha}
$$

which we still may write $\eta_{i}=-\sum_{\lambda} p_{i \lambda}(x) d x_{\lambda}$ with the convention $p_{i n} \equiv-1$.

Lemma 4.1. The integrability conditions may be written locally:

$$
\left(p_{i \lambda}\right)_{\mu}^{\prime}-\left(p_{i \mu}\right)_{\lambda}^{\prime}+p_{i \mu}\left(p_{i \lambda}\right)_{n}^{\prime}-p_{i \lambda}\left(p_{i \mu}\right)_{n}^{\prime} \equiv 0 \text { for all triples }(i, \lambda, \mu) .
$$

Proof. Let $\eta=-\sum_{\lambda} p_{\lambda}(x) d x_{\lambda}$ be a holomorphic 1-form. Then

$$
\begin{array}{r}
\eta \wedge d \eta=\sum_{\lambda<\mu<\nu}\left[p_{\lambda}\left(\left(p_{\nu}\right)_{\mu}^{\prime}-\left(p_{\mu}\right)_{\nu}^{\prime}\right)+p_{\mu}\left(\left(p_{\lambda}\right)_{\nu}^{\prime}-\left(p_{v}\right)_{\lambda}^{\prime}\right)\right. \\
\left.+p_{\nu}\left(\left(p_{\mu}\right)_{\lambda}^{\prime}-\left(p_{\lambda}\right)_{\mu}^{\prime}\right)\right] d x_{\lambda} \wedge d x_{\mu} \wedge d x_{v}
\end{array}
$$

Then, when $p_{n} \equiv-1$, we observe that the vanishing of all terms in $d x_{\lambda} \wedge d x_{\mu} \wedge d x_{n}$ implies the vanishing of all other terms, hence the lemma.

A section $\left(\omega_{i}\right)_{i}$ of $A$ is locally given by the $d$ functions $f_{i}$ such that $\omega_{i}=f_{i}\left(\sum_{\lambda} p_{i \lambda}(x) d x_{\lambda}\right)$ satisfying to the identities

$$
\sum_{i} p_{i \lambda} f_{i} \equiv 0 \quad \text { for any } \lambda,
$$

hence, by derivation,

$$
\left(E_{\lambda, \mu}\right) \quad \sum_{i}\left(p_{i \lambda} f_{i}\right)_{\mu}^{\prime} \equiv 0 \quad \text { for any } \lambda, \mu \text {. }
$$

Lemma 4.2. For the family $\left(f_{i}\right)_{i}$ to define an Abelian relation, it is necessary and sufficient that the identities be satisfied

$$
\left(F_{i \alpha}\right) \quad\left(f_{i}\right)_{\alpha}^{\prime} \equiv-\left(f_{i} p_{i \alpha}\right)_{n}^{\prime} \text { for all pairs }(i, \alpha) .
$$

Proof. In fact, a holomorphic 1-form $f\left(\sum_{\lambda} p_{\lambda}(x) d x_{\lambda}\right)$ is closed iff $\left(f p_{\lambda}\right)_{\mu}^{\prime}=\left(f p_{\mu}\right)_{\lambda}^{\prime}$ for all pairs $(\lambda, \mu)$ such that $\lambda<\mu$. But, because of the integrability conditions, it is sufficient that this relation be satisfied when $\mu=n$, for it to be satisfied with all other $\mu$ 's.

Lemma 4.3. When the family $\left(f_{i}\right)_{i}$ defines an Abelian relation, the identities $\left(E_{\lambda, \mu}\right)$ and $\left(E_{\mu, \lambda}\right)$ are the same. 
Proof. In fact, under the assumption, $\left(f_{i} p_{i \lambda}\right)_{\mu}^{\prime} \equiv\left(f_{i} p_{i \mu}\right)_{\lambda}^{\prime}$ for all pairs $(\lambda, \mu)$, hence the lemma by summation with respect to $i$.

The identity $\left(f_{i}\right)_{\alpha}^{\prime} \equiv-\left(f_{i} p_{i \alpha}\right)_{n}^{\prime}$ means that it is sufficient to know the $\left(f_{i}\right)_{n}^{\prime}$ to know the other partial derivatives $\left(f_{i}\right)_{\alpha}^{\prime}$ of a family $\left(f_{i}\right)_{i}$ defining an Abelian relation.

Writing $w_{i}=\left(f_{i}\right)_{n}^{\prime}$, and combining $\left(E_{\lambda, \mu}\right)$ and $\left(F_{i \alpha}\right)$, we deduce:

Corollary 4.4. The elements of $R_{0}$ above a given element $\left(f_{i}\right)_{i}$ in A map bijectively onto the solutions of the linear system $\Sigma_{0}$.

$$
\left(\widetilde{E}_{\lambda, \mu}\right) \quad \sum_{i} p_{i \lambda} p_{i \mu} w_{i} \equiv \sum_{i} f_{i}\left[\left(p_{i \lambda}\right)_{\mu}^{\prime}-p_{i \lambda}\left(p_{i \lambda}\right)_{n}^{\prime}\right]
$$

of c $(n, 2)$ equations $\left(\widetilde{E}_{\lambda, \mu}\right)$ with d unknown $w_{i}$.

Notice that the matrix of the system $\Sigma_{0}$ is the matrix $P_{2}=\left(\left(C_{i L}^{(2)}\right)\right)_{i, L}$ seen in the previous section.

Proof of the Lemma 3.1 in the case $d<\left(c(n, 2)\right.$. Generically, the system $\Sigma_{0}$ has rank $d$. Hence $S$ is defined locally by the vanishing of all characteristic determinants which are generically not all identically zero. If $\Sigma_{0}$ has a rank $r$ smaller than $d$, the vanishing of all determinants of size $r+1, \cdots, d$ in the matrix $P_{2}$ have to be added to the vanishing of all charateristic determinants.

\section{Computation of $R_{k}(k \geq 1)$}

For any pair of multi-indices of derivation $L=\left(\lambda_{1}, \cdots, \lambda_{s}, \cdots, \lambda_{n}\right)$, and $H=\left(h_{1}, h_{2}, \cdots, h_{n}\right), L+H$ will denote the multi-index $\left(\lambda_{1}+h_{1}, \lambda_{2}+h_{2}, \cdots, \lambda_{n}+h_{n}\right)$. We define similarly $L-H$ if $\lambda_{\mu} \geq h_{\mu}$ for all $\mu$ 's. For any $\lambda, 1_{\lambda}$ will denote the muti-index with all $\lambda_{\mu}$ 's equal to zero for $\mu \neq \lambda$ and $\lambda_{\lambda}=1$. By definition the height $|L|$ of $L$ is the sum $\sum_{s} \lambda_{s}$.

By derivation of the identities $\left(E_{\lambda}\right)$, the elements of $J^{k} A$ are characterized by the identities

$\left(E_{\lambda, L}\right) \quad \sum_{i}\left(p_{i \lambda} f_{i}\right)_{L}^{\prime} \equiv 0$ for any $\lambda$ and for any multi-index $L$ of height $|L| \leq k$.

Lemma 5.1. If $\left(f_{i}\right)_{i}$ is an Abelian relation, the relation $\left(E_{\lambda, L}\right)$ remains unchanged by permutation of all the indices of $L \cup\{\lambda\}$.

Proof. The left hand term of this identity is obviously symetric with respect to the indices of $L$. Thus, it is sufficient to prove that the identities $\left(E_{\lambda, \mu}\right)$ and $\left(E_{\mu, \lambda}\right)$ are the same, which we know already.

The identity $\left(E_{\lambda, L}\right)$ above will now be denoted by $\left(E_{H}\right)$, where $H=L+1_{\lambda}$. 


\section{Lemma 5.2.}

(i) If $\left(f_{i}\right)_{i}$ is an Abelian relation, all partial derivatives $\left(f_{i}\right)_{L}^{\prime}$ may be written as a linear combination

$$
\widetilde{\left(f_{i}\right)_{L}^{\prime}} \equiv \sum_{k=0}^{|L|} D_{i L}^{(k)} \cdot\left(f_{i}\right)_{n^{k}}^{\prime}
$$

of $f_{i}$ and of its partial derivatives $\left(f_{i}\right)_{n^{k}}^{\prime}=\frac{\partial^{(k)} f_{i}}{\left(\partial x_{n}\right)^{k}}$ with respect to the only variable $x_{n}$, with coefficients $D_{i L}^{(k)}$ not depending on the $f_{i}$ 's.

(ii) If $L=\left(\lambda_{1}, \cdots, \lambda_{s}, \cdots, \lambda_{n}\right)$, the coefficient $D_{i L}^{(|L|)}$ of highest order is equal to $(-1)^{|L|} \prod_{s=1}^{n} p_{i}^{\lambda_{s}}$, i.e. is equal to $(-1)^{|L|} C_{i L}^{(|L|)}$.

Proof. We get the lemma by derivation of the identities $\left(F_{i \alpha}\right)$ and an obvious induction on the height $|L|$ of $L$.

The lemma above means that it is sufficient to know the $\left(f_{i}\right)_{n^{k+1}}^{\prime}$ to know the other partial derivatives $\left(f_{i}\right)_{L}^{\prime}$ in the $(k+1)$-jet of a family $\left(f_{i}\right)_{i}$ defining an Abelian relation.

Hence, writing $w_{i}=\left(f_{i}\right)_{n^{k+1}}^{\prime}$, and combining $\left(E_{L}\right.$ and $\left(\widetilde{F}_{i L}\right)$, we get:

Corollary 5.3. The elements of $R_{k}$ above a given element $a_{0}^{(k-1)}$ in $R_{k-1}$ map bijectively onto the solutions of a linear system $\Sigma_{k}$ of $c(n, k+2)$ equations $\left(\widetilde{E}_{L}\right)$ with d unknown $w_{i}$

$$
\sum_{i} C_{i L}^{(k+2)} w_{i} \equiv \Phi_{L}\left(a_{0}^{(k-1)}\right)
$$

where $L$ runs through the set $\mathcal{P}(n, k+2)$ of partitions of $k+2$, and where the second member $\Phi_{L}\left(a_{0}^{(k-1)}\right)$ depends only on $a_{0}^{(k-1)} \in R_{k-1}$. In particular, the symbol $\sigma_{k}$ of $D_{k}$ is defined by the matrix

$$
P_{k+2}=\left(\left(C_{i L}^{|L|}\right)\right)_{i, L},
$$

$1 \leq i \leq d,|L|=k+2$, of size $d \times c(n, k+2)$.

Theorem 5.4. Assume the $d$-web to be ordinary. The map $\pi_{k}: R_{k} \rightarrow R_{k-1}$ is surjective for $k \leq k_{0}-2$ and injective for $k>k_{0}-2$ above the open set $U=M_{0} \backslash S$ of $M_{0}$. For $k \leq k_{0}-2, R_{k}$ is a holomorphic bundle of rank $\sum_{h=1}^{k+2}(d-c(n, h))$ over $M_{0} \backslash S$.

Proof. In fact, $P_{k+2}$ is precisely the matrix of the system $\Sigma_{k}$. Thus, for $k \leq k_{0}-2$ and off $S_{k}$, the space of solutions of $\Sigma_{k}$ is an affine space of dimension $d-c(n, k+2)$. 
Proof of Theorem 1.1 in the case $d>c(n, 2)$. The rank of a ordinary $d$-web is at most equal to the number

$$
\pi^{\prime}(n, d)=\sum_{h=1}^{k_{0}}(d-c(n, h)) .
$$

For $k>k_{0}-2$, the symbol $\sigma_{k}$ is necessarily injective off $S$. In fact, the matrix defining this symbol in the corollary above contains the matrix defining $\sigma_{k-1}$ when we choose the coordinates and the forms $\eta_{i}$ 's so that $p_{i n} \equiv-1$. Hence, since $g_{k_{0}-2}=0$ off $S, g_{k}=0$ off $S$ for all $k \geq k_{0}-2$. Consequently, above a given element in $R_{k_{0}-2}$, there exists at most one infinite jet of Abelian relation, hence one germ of Abelian relation since the framework is analytic. We deduce that the rank of the web is at most $\pi^{\prime}(n, d)$ off $S$, hence everywhere (semi-continuity of the rank). We shall see in the next section that any ordinary SGP parallel web has rank $\pi^{\prime}(n, d)$, hence the optimality.

Proof of Theorem 1.2. When $d=c\left(n, k_{0}\right), \mathcal{E}=\left.R_{k_{0}-3}\right|_{M_{0} \backslash S}$ is a vector bundle of rank $\pi^{\prime}(n, d)$, and $\pi_{k_{0}-2}: R_{k_{0}-2} \rightarrow R_{k_{0}-3}$ is an isomorphism of vector bundles over $M_{0} \backslash S$. Therefore, we just have to use lemma 2-2. In particular, for $d=c\left(n, k_{0}\right)$, the rank of a ordinary $d$-web is $\pi^{\prime}(n, d)$ if and only if the curvature of the previous connexion vanishes.

\section{Examples}

\subsection{Case $n=2$}

We recover the results of [9]. In fact, in this case:

- the set $S$ is always empty (all determinants occuring in the computation of the symbols $\sigma_{k}$ are determinants of Vandermonde for $k \leq k_{0}-2$, vanishing nowhere on $\left.M_{0}\right)$; thus, all webs are ordinary.

- any $d$ is equal to $c(2, d-1)$,

- the rank $\sum_{h=1}^{d-2}(c(2, d-1)-c(2, h))$ of $R_{d-4}$ is equal to $(d-1)(d-2) / 2$.

\subsection{Case $n=3, d=6$}

Use coordinates $x, y, z$ on $\mathbb{C}^{3}$, with $n=3$, and $d=6(=c(n, 2))$. Let $a, b, c, e, h$ be five distinct complex numbers, all different of 0 , and let $\psi$ be some holomorphic function of $y$. Let $W$ be the 6-web of codimension 1 on $\mathbb{C}^{3}$ defined by the 1 -forms $\eta_{i}=d z-p_{i} d x-q_{i} d y, 1 \leq i \leq 6$, where: $\left(p_{1}, q_{1}\right)=(0,0),\left(p_{2}, q_{2}\right)=\left(a, a^{2}\right)$, $\left(p_{3}, q_{3}\right)=\left(b, b^{2}\right),\left(p_{4}, q_{4}\right)=\left(c, c^{2}\right),\left(p_{5}, q_{5}\right)=\left(e, e^{2}\right)$ and $\left(p_{6}, q_{6}\right)=(h, \psi)$. 
The system $\Sigma_{0}$ is then equivalent to

$$
\left(\begin{array}{cccc}
a & b & c & e \\
a^{2} & b^{2} & c^{2} & e^{2} \\
a^{3} & b^{3} & c^{3} & e^{3} \\
a^{4} & b^{4} & c^{4} & e^{4}
\end{array}\right)\left(\begin{array}{l}
w_{2} \\
w_{3} \\
w_{4} \\
w_{5}
\end{array}\right)=\left(\begin{array}{c}
0 \\
0 \\
0 \\
f_{6} \cdot \psi^{\prime}
\end{array}\right)
$$

to which we add the equations $\left(h^{2}-\psi\right) w_{6}=0$, and $w_{1}+w_{2}+\cdots+w_{6}=0$. The system is a system of Cramer off the locus $S$ which is

- the surface of equation $\psi(y)=h^{2}$ in general,

- the empty set when $\psi$ is any constant different of $h^{2}$,

- all of $\mathbb{C}^{3}$ (the special case) for $\psi \equiv h^{2}$.

Let's precise the connection and its curvature for $\psi \neq h^{2}$ in the two first cases (the ordinary cases).

A section $\left(f_{i}\right)$ of $A$ is defined by $\left(f_{4}, f_{5}, f_{6}\right)$, since $\left(f_{1}, f_{2}, f_{3}\right)$ can be deduced using the equations $\sum_{i} f_{i}=0, \sum_{i} p_{i} f_{i}=0$ and $\sum_{i} q_{i} f_{i}=0$.

Set $\Delta=a b c e(b-a)(c-a)(e-a)(c-b)(e-b)(e-c)\left(h^{2}-\psi\right), K=\frac{\psi^{\prime}}{\Delta\left(h^{2}-\psi\right)}$, $\Delta_{4}=a b e\left((b-a)(e-a)(e-b)\right.$, and $\Delta_{5}=a b c((b-a)(c-a)(c-b)$. We then get: $w_{4}=-K \Delta_{4}, w_{5}=K \Delta_{5}, w_{6}=0$, hence $u_{4}=c K \Delta_{4}, u_{5}=-e K \Delta_{5}, u_{6}=0$ and $v_{4}=c^{2} K \Delta_{4}, u_{5}=-e^{2} K \Delta_{5}, u_{6}=0$. With respect to the trivialization $\left(\sigma_{4}, \sigma_{5}, \sigma_{6}\right)$ of $A$ given by $\sigma_{4}=\left(f_{4} \equiv 1, f_{5} \equiv 0, f_{6} \equiv 0\right), \sigma_{5}=\left(f_{4} \equiv 0, f_{5} \equiv 1, f_{6} \equiv 0\right)$ and $\sigma_{6}=\left(f_{4} \equiv 0, f_{5} \equiv 0, f_{6} \equiv 1\right)$, the matrix of the connection is:

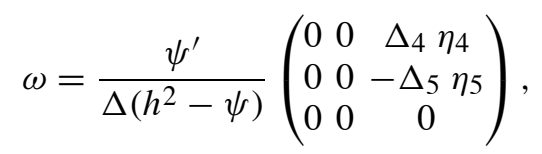

hence the curvature

$$
\Omega=\frac{1}{\Delta}\left(\frac{\psi^{\prime}}{h^{2}-\psi}\right)^{\prime}\left(\begin{array}{ccc}
0 & 0 & \Delta_{4}(d y \wedge d z+c d x \wedge d y) \\
0 & 0 & -\Delta_{5}(d y \wedge d z+e d x \wedge d y) \\
0 & 0 & 0
\end{array}\right)
$$

We observe that $\sigma_{4}$ and $\sigma_{5}$ are linearly independant Abelian relations. We knew it already since the first integrals $\left(z-c x-c^{2} y\right)$ and $\left(z-e x-e^{2} y\right)$ of $\mathcal{F}_{4}$ and $\mathcal{F}_{5}$ respectively are linear combinations of the first integrals $z,\left(z-a x-a^{2} y\right)$ and $\left(z-b x-b^{2} y\right)$ of $\mathcal{F}_{1}, \mathcal{F}_{2}$ and $\mathcal{F}_{3}$. Thus, when $h^{2}-\psi$ does not vanish, the rank of the 6 -web is at least 2 , and has the maximum possible value $\pi^{\prime}(3,6)=3$ in the ordinary case if and only if $\frac{\psi^{\prime}}{h^{2}-\psi}$ is constant, that is if there exists two scalar constant $C$ and $D(C \neq 0)$, such that

$$
\psi(y)=h^{2}+C e^{D y},
$$

in particular for $\psi=$ constant (case $D=0$ ). For given $C$ and $D$ as above, $K=-D / \Delta$, and $\sigma_{6}-K \Delta_{4} \sigma_{4}+K \Delta_{5} \sigma_{5}$ is an Abelian relation (the function $\psi$ occurs in the computation of $f_{1}, f_{2}$ and $f_{3}$ from $f_{4}=-K \Delta_{4}, f_{5}=K \Delta_{5}$ and $\left.f_{6}=1\right)$.

The special case $\psi \equiv h^{2}$ will be seen in the next subsection. 


\subsection{Ordinary parallel webs and optimality of the bound $\pi^{\prime}(n, d)$}

For any pair $(n, d)$ with $d>n$, give $d$ linear forms $\left(l_{1}, l_{2}, \cdots, l_{d}\right)$. Let $\mathcal{F}_{i}$ be the foliation defined in $\mathbb{C}^{n}$ by the parallel hyperplanes $l_{i}=$ constant, and $W$ be the $d$-web defined by these $d$ foliations. Let $k_{0}$ be the integer such that $c\left(n, k_{0}\right) \leq d<c\left(n, k_{0}+1\right)$. Let $\mathbb{P}_{n-1}$ denote the hyperplane at infinity of the $n$-dimensional projective space $\mathbb{P}_{n}=\mathbb{C}^{n} \coprod \mathbb{P}_{n-1}$ : the parallel hyperplanes of the pencil $l_{i}=$ constant meet at infinity along a hyperplane of $\mathbb{P}_{n-1}$, i.e. define an element $\left[l_{i}\right]$ of the dual projective space $\mathbb{P}_{n-1}^{\prime}$.

Remark 6.1. The web $W$ on $\mathbb{C}^{n}$ extends to a web on $\mathbb{P}_{n}$ (with singularities) which is algebraic. It is in fact dual to the union of $d$ straight lines in the dual projective space $\mathbb{P}_{n}^{\prime}$.

Definition 6.2. Such a web on $\mathbb{C}^{n}$ will be said a parallel $d$-web.

Lemma 6.3. The two following properties are equivalent:

(i) The parallel $d$-web above is ordinary.

(ii) For any $h,\left(1 \leq h \leq k_{0}\right)$, there exists $c(n, h)$ points among the $d$ points $\left[l_{i}\right]$, which do not belong to a same algebraic (reducible or not) hypersurface of degree $h$ in $\mathbb{P}_{n-1}^{\prime}$.

Proof. For $d \geq c(n, h),(h \geq 1)$, assume that the matrix $\left(\left(C_{i L}^{(h)}\right)\right)_{i, L}, 1 \leq i \leq d$, $|L|=h$, of size $d \times c(n, h)$ has rank $<c(n, h)$. This means that the determinant of any square sub-matrix of size $c(n, h)$ vanishes. Saying that the determinant of the sub-matrix given for instance by the $c(n, h)$ first $l_{i}$ 's vanishes means precisely that $\left[l_{1}\right],\left[l_{2}\right], \cdots,\left[l_{c(n, h)}\right]$ belong to some hypersurface of degree $h$ in $\mathbb{P}_{n-1}^{\prime}$ (may be reducible), and same thing for any other subset of $c(n, h)$ indices $i$. Hence the lemma is proved.

Corollary 6.4. The bound $\pi^{\prime}(n, d)$ for the rank of an ordinary web is optimal.

Proof. Remember that an algebraic hypersurface of degree $h$ in $\mathbb{P}_{n-1}^{\prime}$ is defined in general by the data of $c(n, h)-1$ of its points: thus, the property (ii) of Lemma 6.3 above is generically satisfied, so that there exists (many) ordinary parallel $d$-webs in dimension $n$ for any $(n, d)$.

By contraposition of the previous lemma, we get also that special parallel $d$ webs are defined by $d$ points $\left[l_{i}\right]$ in $\mathbb{P}_{n-1}^{\prime}$ belonging to a same algebraic hypersurface of degree $h$ in $\mathbb{P}_{n-1}^{\prime}$, for some $h,\left(1 \leq h \leq k_{0}\right)$.

\section{Theorem 6.5.}

(i) If the $d$ points $\left[l_{i}\right]$ are in general position in $\mathbb{P}_{n-1}^{\prime}$ (i.e. if any $n$ of the $d$ linear forms $l_{i}$ are linearly independant), the above parallel $d$-web has rank $\geq \pi^{\prime}(n, d)$.

(ii) It has exactly rank $\pi^{\prime}(n, d)$ if and only if it is ordinary. 
Proof. For any $h$, consider the vector space $L_{h}$ generated by the $h^{\text {th }}$ symetric products $\left(l_{i}\right)^{h}$ of the $l_{i}$ 's, and denote by $r_{h}$ the dimension of this vector space. After [19], the rank of a parallel SGP web is $\sum_{h=1}^{k_{0}}\left(d-r_{h}\right)$. Hence we have only to prove that $r_{h} \leq c(n, h)$ in general, and $r_{h}=c(n, h)$ in the ordinary case. But this is obvious after the previous lemma, since the dimension of $L_{h}$ is exactly the rank of the matrix $P_{h}=\left(\left(C_{i L}^{(h)}\right)\right)_{i, L}$ with $i \leq d,|L|=h$ in the linear system $\Sigma_{h-2}$ of Corollary 5.3.

For instance, a parallel SGP 6-web on $\mathbb{P}_{3}$ will be special of rank $4(=\pi(3,6))$ or ordinary of rank $3\left(=\pi^{\prime}(3,6)\right)$, according to the fact that the six points $\left[l_{i}\right]$ belong or not to a same conic of $\mathbb{P}_{2}^{\prime}$ : when $\psi$ is constant in the example $\left(p_{1}, q_{1}\right)=(0,0)$, $\left(p_{2}, q_{2}\right)=\left(a, a^{2}\right),\left(p_{3}, q_{3}\right)=\left(b, b^{2}\right),\left(p_{4}, q_{4}\right)=\left(c, c^{2}\right),\left(p_{5}, q_{5}\right)=\left(e, e^{2}\right)$ and $\left(p_{6}, q_{6}\right)=(h, \psi)$ of the previous subsection, the five first points belong to the conic of equation $q=p^{2}$, hence the dichotomy according to the fact that $\psi$ is equal or different of $h^{2}$. [In the special case, it is amazing to deduce the fourth Abelian relation from the Pascal's theorem of the hexagon.]

New proof of the Theorem 1.1 for $d \geq c(n, 2)$ in the case of SGP ordinary webs. The rank of a SGP web is upper-bounded by the rank of the "tangent parallel web" at a point. Since the fact that a web be ordinary near a point is equivalent to the fact that the tangent parallel web at that point is ordinary, theorem 1-1 is also a corollary of the previous Theorem 6.5, when the ordinary web is SGP.

\subsection{An example of ordinary web of maximal rank 26 for $n=3, d=15$}

The following example has been given to us by L. Pirio ([17]).

In $\mathbb{C}^{3}$ with coordinates $x, y, z$, consider the 15 -web defined by ten pencils of planes, four pencils of quadratic cones or cylinders, and a last pencil of quadrics, respectively defined by the following first integrals $u_{i}(1 \leq i \leq 15)$ :

$$
\begin{aligned}
& u_{1}=x \quad, u_{2}=y \quad, u_{3}=\quad z \text {, } \\
& u_{4}=\frac{z}{z-y} \quad, \quad u_{5}=\frac{z}{z-x} \quad, u_{6}=\frac{y}{y-x}, \\
& u_{7}=\frac{1-z}{y-z} \quad, \quad u_{8}=\frac{1-z}{z-x} \quad, u_{9}=\frac{1-y}{y-x}, \\
& u_{10}=\frac{x-y}{z-y} \quad, u_{11}=\frac{z(1-y)}{z-y}, u_{12}=\frac{z(1-x)}{z-x}, \\
& u_{13}=\frac{y(1-x)}{y-x}, u_{14}=\frac{z(x-y)}{x(z-y)}, u_{15}=\frac{(1-z)(y-x)}{(1-x)(y-z)} .
\end{aligned}
$$

Denote by $m_{X}=[1 ; 0 ; 0 ; 0], m_{Y}=[0 ; 1 ; 0 ; 0], m_{Z}=[0 ; 0 ; 1 ; 0]$ and $m_{T}=[0 ; 0 ; 0 ; 1]$ the vertices of the standard projective frame in $\mathbb{P}_{3}$ : the foliations 1 to 6 are the pencils of planes through the six lines of the tetrahedron. Denote by $\Omega_{X}=[0 ; 1 ; 1 ; 1], \Omega_{Y}=[1 ; 0 ; 1 ; 1], \Omega_{Z}=[1 ; 1 ; 0 ; 1]$ and $\Omega_{T}=[1 ; 1 ; 1 ; 0]$ 
the barycenters of the faces of the previous tetrahedron: the foliations 7, 8, 9 and 10 are respectively the foliations given by the pencil of planes through the line $m_{X} \Omega_{X}$ (respectively $m_{Y} \Omega_{Y}, m_{Z} \Omega_{Z}$ and $m_{T} \Omega_{T}$ ).

The foliation 11 is the pencil of the quadratic cylinders of vertex $m_{X}$ having for base the conics through $\Omega_{X}, m_{Y}, m_{Z}, m_{T}$ in the plane $X=0$. We get similarly the foliations 12,13 , and 14 by permutation of the letters $X, Y, Z, T$.

The 5-subweb $(1,2,3,10,14)$ is then the 5-web of cones of vertex $m_{T}$ above the Bol's 5-web defined by the four points $m_{X}, m_{Y}, m_{Z}, \Omega_{T}$ in the plane at infinity $T=0$. We can do the same with the other 5-subwebs $(2,3,4,7,11),(1,3,5,8,12)$ and $(1,2,6,9,13)$.

L. Pirio proved by computer that this 15 -web is ordinary (see [17]). Pereira and Pirio exhibited directly 26 independant Abelian relations: since $\pi^{\prime}(3,15)=26$, the rank of the web is the biggest possible for ordinary webs (while $\pi(3,15)=42$ ). Observe that $15=c(3,4)$; thus, it would be obviously very interesting to prove directly (may be by computer) the vanishing of the curvature. For the moment, we did not do it, our ability on computer being poor, and the people to whom we asked to do the job saying that it was too complicated.

The previous web corresponds in fact to the particular case $n=3$ of the following example: the group $P G L(1)$ of the automorphisms of the complex projective line $\mathbb{P}_{1}$ acts naturally on the open subset $\left(\mathbb{P}_{1}\right)_{0}^{n+3}$ of $\left(\mathbb{P}_{1}\right)^{n+3}$ of the ordered families of $n+3$ distinct points in $\mathbb{P}_{1}$. Let $M_{0, n+3}$ be the $n$-dimensional quotient manifold $\left(\mathbb{P}_{1}\right)_{0}^{n+3} / P G L(1)$. Any choice of four distinct ordered points in $\mathbb{P}_{1}$, among the $n+3$, defines a forgetful map $M_{0, n+3} \rightarrow M_{0,4}$ (this is the "bi-ratio" of four points, which is preserved by $P G L(1))$, hence the codimension one foliation on $M_{0, n+3}$ by the level hypersurfaces of this map, and a $\left(\begin{array}{c}n+3 \\ 4\end{array}\right)$-web on $M_{0, n+3}$ denoted by $\mathcal{W}\left(A_{0, n+3}\right)$ when taking all possible choices of four points among $n+3$.

J. V. Pereira proved more generally in [15] that, for any $n \geq 2, \mathcal{W}\left(A_{0, n+3}\right)$ is ordinary, and has maximal rank $\pi^{\prime}\left(n,\left(\begin{array}{c}n+3 \\ 4\end{array}\right)\right)$. Since $\left(\begin{array}{c}n+3 \\ 4\end{array}\right)=c(n, 4)$, $\mathcal{W}\left(A_{0, n+3}\right)$ has a curvature, whose vanishing might of course be proved directly. But, even in the well known case $n=2$ of the Bol's web, this is not so easy, hence again the necessity to perform some program for doing it by computer.

\section{References}

[1] W. BlaschKe and G. Bol, "Geometrie der Gewebe, Die Grundlehren der Mathematik", Vol. 49, Springer, Berlin, 1938.

[2] S. S. CHERN, Abzählungen für Gewebe, Abh. Hamburg 11 (1936), 163-170.

[3] S. S. CHERN and P. A. GRIFFITHS, Abel's theorem and webs, Jahresber. Deutsch. Math.Verein. 80 (1978), 13-110 and 83 (1981), 78-83.

[4] V. CAVALIER and D. LEHMANn, Ordinary holomorphic webs of codimension one, preprint, arXiv: math 0703596 v2[mathDS] 13 Oct. 2008. 
[5] V. Cavalier and D. Lehmann, Rang et courbure de Blaschke des tissus holomorphes réguliers de codimension un, C.R. Math. Acad. Sci. Paris 346 (2008), 1283-1288.

[6] V. CAVAliER and D. Lehmann, Global structure of webs in codimension one, preprint, arXiv: math v1 [mathDS] March. 2008.

[7] V. CaVAliER and D. Lehmann, Introduction à l'étude globale des tissus sur une surface holomorphe, Ann. Inst. Fourier (Grenoble) 57 (2007), 1095-1133.

[8] P. A. GRIFFITHS and J. HARRIS, "Principles of Algebraic Geometry", John Wiley \& Sons, New York, 1978.

[9] A. HÉNAUT, On planar web geometry through Abelian relations and connections, Ann. of Math. 159 (2004), 425-445.

[10] A. HÉNAUT, Systèmes différentiels, nombre de Castelnuovo et rang des tissus de $\mathbb{C}^{n}$, Publ. Res. Inst. Math. Sci. 31 (1995), 703-720.

[11] A. HÉnAut, Formes différentielles abéliennes, bornes de Castelnuovo et géométrie des tissus, Comment. Math. Helv. 79 (2004), 25-57.

[12] A. HÉNAUT, Planar web geometry through abelian relations and singularities, In: "Nankai Tracts in Mathematics" P. Griffiths (ed.), Vol. 11, World Scientific and Imperial College Press, 2006.

[13] A. PANTAZI, Sur la détermination du rang d'un tissu plan, C.R. Inst. Sci. Roum. 2 (1938), $108-111$.

[14] J. V. Pereira, Algebrization of codimension one webs [after Trépreau, Hénaut, Pirio, Robert], Séminaire Bourbaki 2006-2007, n. 974, Mars 2007.

[15] J. V. PEREIRA, Resonance webs of hyperplane arrangements, Adv. Stud. Pure Math. 99 (2010), 1-30.

[16] J. V. Pereira and L. Pirio, "An invitation to Web Geometry", 27 Colóquio Brasileiro de Matemática, Publicaões matemáticas do IMPA, Instituto de matemática pura e aplicada, Rio de Janeiro, 2009.

[17] L. PIRIO, Sur la linéarisation des tissus, preprint, arXiv: math 0811.1810v2 [mathDG] 23 janv. 2009.

[18] D. C. SPEnCER, "Selecta", Vol. 3, World Scientific Publishing Co. Philadelphia, 1985.

[19] J. M. Trépreau, Algébrisation des tissus de codimension 1. La généralisation d'un théorème de Bol, Inspired by S. S. Chern, In: "Nankai Tracts in Mathematics", P. Griffiths (ed.), Vol. 11, World Scientific and Imperial College Press, 2006.

4 rue Becagrun 30980 Saint Dionisy, France lehmann@math.univ-montp2.fr 